\title{
Immunotherapy in Breast Cancer - Towards a New Understanding of Both Tumor and Host
}

\author{
Peter C. Dubsky ${ }^{a} \quad$ Giuseppe Curigliano ${ }^{b}$ \\ aDepartment of Surgery, Medical University of Vienna, Austria \\ ${ }^{b}$ Department of Medical Oncology, European Institute of Oncology, Milan, Italy
}

The basic concept of immunotherapy in cancer is to enable the immune system to detect neoplastic growth and to either prevent carcinogenesis and/or reject transformed cells with a potential for malignant tumor growth. Taking into account the enormous success of vaccines against infectious diseases, such an approach has the potential of not only successfully treating cancer patients but also preventing recurrences for an extended period of time.

The approach also has a particular appeal to patients (and their physicians). The activation of an intrinsic system to reject what is considered as pathologic is very close to what most patients intuitively seek as they strive for a cure or a process of healing. Also, side effects (especially in comparison to cytotoxic drugs) are believed to be limited.

Immunotherapy has become a clinical reality for a number of both hematological malignancies and solid organ cancers [1]. However, with the exception of antibody-based HER2 targeting, immunotherapy in breast cancer and especially the implementation into clinical trials has largely been a frustrating experience over the last two decades. So why, in view of many unsuccessful trials, should immunotherapy have a role in breast cancer? In this issue of BREAST CARE we review recent advances that lead to a new understanding of not only cancer immunology but also of breast cancer as a heterogeneous disease:

Stagg and colleagues [2] review how chemotherapy and targeted treatments modulate the immune system. Immune response to cancer is a dynamic process that can lead to the rejection of cancer but can also have regulatory effects that promote tumor growth. This concept of immunoediting in cancer immunology has profound impact on how we understand some of the long-term efficacy of chemotherapy and radiotherapy today; treatments formerly believed to be highly immunosuppressive.

Sheeba Irshad and her colleagues [3] from Guy's hospital demonstrate how advances in genomic sequencing technologies have stratified breast cancer subtypes using immune- related gene signatures. Using gene profiling we learn how intrinsic subtypes of breast cancer may differ in their immunogenicity and as a consequence how host-tumor interaction may be altered using both conventional or immunotherapeutic treatments.

Two up-to-date reviews, both from the European Institute of Oncology in Milan [4, 5], focus on novel advances in the identification of tumor associated antigens in breast cancer and the challenge to overcome the 'self' nature of some of these epitopes in order to develop vaccines for cancer prevention.

In summary, this issue highlights how research from two main directions has changed both our ideas and our future direction in implementing immunotherapies into the clinic: The recognition of breast cancer heterogeneity and the qualitative differences in tumor biology help to focus immune related therapies to entities that have a fair chance of treatment response. Novel immunotherapies are a consequence of several paradigm shifts concerning our understanding of the immune response (or the lack thereof) to cancer.

Currently, several phase I-II clinical trials are underway to investigate immunotherapeutic approaches. Increasingly, these trials try to focus on early breast cancer in order to better overcome set regulatory pathways of the immune response. These trials also have in common that they stratify or select women according to intrinsic subtypes. It is likely that the combined understanding of tumor and host response may lead to an extension of the immunotherapeutic advances to breast cancer.

The identification of immunological and genetic features affecting immune response in patients with minimal tumor burden is the optimal background for development of clinical studies in the adjuvant setting. An active immunization has the potential advantages of a non-toxic therapeutic modality capable of inducing antitumor immune responses in patients with tumors. Induction of strong immunity by cancer vaccines is expected to lead to the establishment of immunological

\section{KARGER}

Fax +497614520714

Information@Karger.de

www.karger.com (c) 2012 S. Karger GmbH, Freiburg

$1661-3791 / 12 / 0074-0258 \$ 38.00 / 0$

Accessible online at:

www.karger.com/brc
Peter C. Dubsky, MD

Department of Surgery

Medical University of Vienna

Währinger Gürtel 18-20, 1090 Vienna, Austria

Tel. +43 1 40400-5621, Fax -6574

peter.dubsky@meduniwien.ac.at 
memory, thereby preventing tumor recurrence. In order to optimize the immunological response to a vaccination strategy, we need first to identify the target antigen and the patient population to be targeted. Research on tumor-associated antigens (TAAs) has identified a large collection of peptide epitopes that have been and are being used for vaccination of cancer patients [5]. Several potential advantages of using peptide-based vaccines include i) easy and relatively inexpensive production of synthetic peptides; ii) easy administration of peptides in a clinical setting; iii) possibility of treating only those patients whose tumors overexpress the target antigens and iv) availability of in vitro or ex vivo assays that can assess patients' immune response to vaccine epitopes [5]. These antigens are down-regulated in somatic adult tissues while become aberrantly re-expressed in various malignancies. TAA expression is associated with a poorer outcome and is more prevalent in higher-grade and advanced-stage tumors. An intensive research into their possible use as therapeutic vaccines is actually ongoing due to their potent immunogenicity.

The aim of future studies will be to assess the immunoreactivity of several antigens in a large series of breast cancer samples classified according to molecular subtypes. Identification of potential target in subpopulations of patients with breast cancer may allow identification of patients who are potential candidates for adjuvant therapeutic vaccines. It is our current thinking that patients with minimal residual disease after preoperative chemotherapy are the ideal setting to test the efficacy of a vaccination strategy. To date, vaccines for breast cancer have been mainly used in end-stage disease. Several clinical studies have been completed with vaccines against antigens such as MUC1, CEA, HER2 and the carbohydrate antigens with varying results [5]. TAA antigens offer a novel opportunity for fostering vaccine development and therapy.

An ideal and successful vaccine should have: a target antigen on tumor cells to direct the immune response; a platform to present the vaccine-derived antigen to the immune system; an adjuvant to enhance immune stimulation; and appropriate monitoring techniques. The design of a clinical trial in patients with breast cancer should identify the better population candidate to a vaccine trial and, primarily, the better antigen. Large population analyses on specific subtypes of breast cancer are necessary in order to select patients who have higher probability to express that specific antigen. First select the patient, then drive the design of the clinical trial! In order to design 'second generation' immunotherapy protocols, we should highlight 3 issues: i) the ability to initiate tumor-specific immunity, either directly by providing tumor-associated antigens or indirectly by favoring the cross-presentation of endogenous tumor antigens; ii) the capacity to recruit effector immune cells within the tumor site, by increasing tumor visibility; iii) the ability to preserve immune cell functionality within the tumor microenvironment through the subversion of immune escape mechanisms. It is becoming clear that these three features cannot be provided for by a single modality, and combined therapies should be proposed. Prediction of clinical efficacy based on immunologic monitoring is crucial for the rational design of cancer vaccination studies. Doses, immunization schedules, methods of administration as well as timing of vaccinations and of following boosts to maintain a durable immune response need to be addressed in prospective clinical trials. Optimal combination vaccine therapy with a variety of novel approaches (e.g., monoclonal antibodies such as trastuzumab or tyrosine kinase inhibitors) is a great promise but it also requires evaluation in clinical trials to assess its benefit. More focused developmental guidelines are needed to address characteristics of therapeutic cancer vaccines.

Several questions are raised by the remarkable data presented in this issue of BREAST CARE; answer to these questions should be considered possible area of research in the following years: Should all cancer patients be treated with an active immunotherapy approach or only individuals potentially more 'responding'? How can we predict that the individual will develop an immune response against a particular antigen used in the vaccine formulation? Is there any genetic signature predicting response to immunotherapy? What are the risks associated with such a vaccination, i.e., the possibility to develop an autoimmune response? What is the durability of immune protection? Can we combine vaccine therapy with therapeutic monoclonal antibodies or small target oriented molecules? Continued basic research into the molecular mechanisms regulating carcinogenesis and immunosurveillance/tolerance will identify new potential targets introducing vaccine therapy in prevention trials for patients at high risk for developing cancer. Target antigens that are directly involved in promoting the neoplastic process can induce an optimal antibody response. During tumor progression, several genetic hits might make specific signaling pathways redundant and relevant for activating other downstream pathways. As preventive vaccines operate during the early phases of carcinogenesis, effective inhibition of the specific targets will arrest the whole. As for all preventive medicine, an extremely low incidence of adverse effects will be a prerequisite of preventive cancer vaccines. Another important long-term concern for cancer preventive vaccination is the induction of autoimmunity, which depends on the kind of tumor antigen that is targeted and the response that is elicited. Induction of a specific immune response against the most common oncoantigens overexpressed by pre-neoplastic lesions might constitute a new scenario in cancer prevention. The translation of preclinical data into preventive treatments requires more attention since the plan is to vaccine a healthy individual. Vaccination against selected oncoantigens of healthy people who have a specific genetic risk of cancer, who have been exposed to an exogenous carcinogen, or who bear multifocal pre-neoplastic lesions would provide the most appropriate scenario [4]. There is no doubt that the findings reported in cancer preven- 
tion vaccination trials open a new field at the interface of basic science, clinical medicine, public health, and public policy. It is important to keep in mind that these new treatments raise many scientific, medical, economic, and sociological questions. To improve the efficacy of the breast cancer vaccines, we need a better understanding of the relation between innate and adaptive immune responses, and of the immune escape mechanisms employed by tumor cells, the discovery of mechanisms underlying immunological tolerance, and acknowledgment of the importance of both cell-mediated and humoral adaptive immunity for the control of tumor growth.

Cancer takes advantage of this ability to hide from the immune system by exploiting a series of immune escape mechanisms that were developed to avoid autoimmunity (mechanisms of tolerance). Among these mechanisms are the hijacking of immune-cell-intrinsic checkpoints that are induced on T-cell activation. Blockade of one of these checkpoints, cytotoxic T-lymphocyte-associated antigen 4 (CTLA4) [6] or the programmed death 1 (PD-1) receptor [7-8], recently provided the first evidence of activity of an immune modulation approach in the treatment of a solid tumor. The future frontier in the treatment of cancer requires identification of potential targets in order to personalize therapies. The immune system remembers what it targets, so once the system is correctly activated, it may mediate a durable tumor response.

\section{Disclosure Statement}

The authors declared no conflict of interest.

\section{References}

1 Mellman I, Coukos G, Dranoff G: Cancer immunotherapy comes of age. Nature 2011;480:480-489.

2 Stagg J, Andre F, Loi S: Immunomodulation via chemotherapy and targeted therapy: a new paradigm in breast cancer therapy? Breast Care 2012;7 DOI: $10.1159 / 000342166$.

3 Irshad S, Grigoriadis A, Lawler K, Ng T, Tutt A: Profiling the immune stromal interface in breast cancer and its potential for clinical impact. Breast Care 2012;7: DOI: 10.1159/000341529.
4 Lazzeroni M, Serrano D: Potential use of vaccines in the primary prevention of breast cancer in high-risk patients. Breast Care 2012;7: DOI: $10.1159 / 000342167$.

5 Criscitiello C: Tumor-associated antigens in breast cancer. Breast Care 2012;7: DOI: $10.1159 / 000342164$

6 Robert C, Thomas L, Bondarenko I, et al: Ipilimumab plus dacarbazine for previously untreated metastatic melanoma. N Engl J Med 2011;364:2517-2526.
7 Topalian SL, Hodi FS, Brahmer JR, et al: Safety, activity, and immune correlates of anti-PD-1 antibody in cancer. N Engl J Med 2012;366:2443-2454. 8 Brahmer JR, Tykodi SS, Chow LQM, et al: Safety and activity of anti-PD-L1 antibody in patients with advanced cancer. N Engl J Med 2012;366:24552465. 